\title{
COMPUTER TRANSFORMATION OF DIGITAL IMAGES AND PATTERNS
}




\section{World Scientific Series in Computer Science}

\section{Published}

Volume 1: Computer-aided Specification Techniques

by J Demetrovics, E Knuth \& P Rado

Volume 2: Proceedings of the 2nd RIKEN International Symposium on Symbolic and Algebraic Computation by Computers edited by $N$ Inada \& T Soma

Volume 3: Computational Studies of the Most Frequent Chinese Words and Sounds by Ching $Y$ Suen

Volume 4: Understanding and Learning Statistics by Computer by $M C K$ Yang \& $D H$ Robinson

Volume 5: Visualizing Abstract Objects and Relations - A Constraint-Based Approach by $T$ Kamada

Volume 6: DC Flux Parametron

A New Approach to Josephson Junction Logic

by $E$ Goto \& KF Loe

Volume 8: Information, Randomness \& Incompleteness - Papers on Algorithmic Information Theory by $G J$ Chaitin

Volume 9: P-Prolog - A Parallel Logic Programming Language by Rong Yang

Volume 10: Intelligent Chinese Language Pattern and Speech Processing edited by P S.P Wang

Volume 11: Control Theory of Robotic Systems by JM Skowronski

Volume 12: An Introduction to Chinese, Japanese, and Korean Computing by JK T Huang \& T D Huang

Volume 13: Mathematical Logic for Computer Science by $Z W L u$

Volume 14: Computer Vision and Shape Recognition edited by A Krzyzak, T Kasvand \& C Y Suen

Volume 15: Stochastic Complexity in Statistical Inquiry by J Rissanen

Volume 16: A Perspective in Theoretical Computer Science - Commemorative Volume for Gift Siromoney edited by $R$ Narasimhan

Volume 17: Computer Transformation of Digital Images and Patterns by ZCLi, TD Bui, YY Tang \& CY Suen

\section{Forthcoming}

Volume 7: Syntactic and Structural Pattern Recognition - Theory and Applications edited by $H$ Bunke \& A Sanfeliu

Volume 18: Array Grammars, Patterns and Recognizers edited by P S P Wang

Volume 19: Structural Pattern Analysis edited by $R$ Mohr, Th Pavlidis \& A Sanfeliu 
Series in Computer Science - Vol. 17

\title{
COMPUTER TRANSFORMATION OF DIGITAL IMAGES AND PATTERNS
}

\author{
ZI CAI LI \\ TIEN DAI BUI \\ YUAN YAN TANG \\ CHING Y. SUEN \\ Centre de recherche informatique de Montréal \\ and \\ Department of Computer Science \\ Centre for Pattern Recognition and Machine Intelligence \\ Concordia University \\ Montréal, Québec H3G 1M8, Canada
}




\section{Published by}

World Scientific Publishing Co. Pte. Ltd., P O Box 128, Farrer Road, Singapore 9128

USA office: 687 Hartwell Street, Teaneck, NJ 07666

UK office: 73 Lynton Mead, Totteridge, London N20 8DH

Library of Congress Cataloging-in-Publication data is available.

\section{COMPUTER TRANSFORMATION OF DIGITAL IMAGES AND PATTERNS}

Copyright $\odot 1989$ by World Scientific Publishing Co. Pte. Ltd.

All rights reserved. This book, or parts thereof, may not be reproduced in any form or by any means, electronic or mechanical, including photocopying, recording or any information storage and retrieval system now known or to be invented, without written permission from the Publisher.

ISBN $9971-50-951-2$

Printed in Singapore by JBW Printers \& Binders Pte. Ltd. 
獻給我㑚的雙親

Kính dâng hương hồn Thằy
Me với hết lòng quí mến cuảa con

A Nos Parents

To Our Parents 
This page is intentionally left blank 


\section{Preface}

This book deals with the subject of geometric transformations of digitized images and patterns by computers to develop systematically various shape models and discrete techniques, in particular for picture processing and pattern recognition. Transformations discussed in this book include linear, quadratic, cubic, bilinear, biquadratic, bi-cubic, Coons models and other nonlinear forms such as harmonic and perspective transformations. Since they concern only the geometrical shapes and geometrical properties of the images or patterns, they are commonly known as geometric transformations, which can be used in computer graphics, character recognition, pattern recognition, computer vision and image processing.

Computer graphics is one of the most exciting and rapidly growing fields. As usual, displayed objects are defined by sets of coordinate points. Hence, geometric transformations are procedures for calculating new coordinate positions of these points, as required by a specified change in size and orientation of the object. Most books on computer graphics cover only the basic transformations such as translation, scaling, rotation, and reflection. To achieve more complicated forms and shapes, we need other kinds of geometric transformations and efficient algorithms to carry them out without creating unacceptable errors and deviations. Also efficient discrete techniques have been exploited in this book to facilitate digital images and patterns through the geometric transformations and to avoid the superfluous holes or "measles".

In pattern and character recognition, one of the most interesting and challenging problems is the recognition of characters and patterns which are subjected to "distortions". For instance, distortions result from handwritten characters or objects scanned at an oblique 
angle. Since only the standard images are ready to be recognized by computers, distorted images require "normalization" using inverse transformations. Nevertheless, by using all existing approaches, inverse transformations are difficult to realize due to the necessity of solving nonlinear equations which often produce multiple solutions. In this book we will provide new efficient methods to carry out inverse transformations which bypass the need to solve nonlinear equations.

Since three dimensional (3D) computer vision requires the analysis of $2 \mathrm{D}$ perspective views, perspective transformations are also introduced to analyze inverse perspective transformations which are used to normalize $2 \mathrm{D}$ images.

In this book, we will present a number of useful transformation models in Chapters 2, 3 and 5, provide efficient discrete techniques for image conversion in Chapter 4 , and apply both models and techniques to the recognition of geometrical shapes, Roman letters and Chinese characters in Chapter 6.

It is worth pointing out that the materials in Chapters $4-6$ have been largely selected from our recent research work, on which detailed reports can be found in some papers published in conference proceedings and journals. Furthermore, the methodology in this book for image processing and pattern recognition is mainly based on classical mathematics (e.g., calculus) and numerical methods (e.g., approximate integration). Since the approaches and results represented are new, we provide rather detailed numerical and graphical examples to verify the analysis and to show the promising applications. We therefore hope that such a style would make this book vivid and readable, and that this book would stimulate further research in the related fields.

Montréal

July 1989
Zi Cai Li

in collaboration with

Tien Dai Bui

Yuan Yan Tang

Ching Y. Suen 


\section{Acknowledgments}

We wish to express our gratitude to Mr. Quan-Lin Gu, Senior Engineer of Jiangsu Research Institute of Computing Technology, Nanjing, China, for contributing to some of our research projects described in this book during his visit at Concordia University. We are indebted to $\mathrm{Mr}$. George Zixi $\mathrm{Li}$ for formatting and typing of this manuscript.

The Centre de recherche informatique de Montréal (CRIM) provided ample computer facilities to produce this book. We wish to thank Dr. Pierre Coulombe, Dr. Jacqueline Bourdeau, Mr. Daniel Gorham, Mr. Michel Savoie and others at CRIM for their strong support and assistance during our research and writing processes, and especially to $\mathrm{Mr}$. Charles Snow for his valuable assistance in document preparation of this book.

We are grateful to the staff of World Scientific Publishing Co. for their collaboration to bring this book to fruition. We also thank our families for their enthusiastic support during the writing period of this book. The first author wishes to mention special thanks to Mr. John Fraser for his great help, support and encouragement offered to him.

This book and its related research work are supported in part by the Natural Sciences and Engineering Research Council of Canada, by the Fonds pour la Formation de Chercheurs et l'Aide à la Recherche of Québec, and by the Ministère de l'Enseignement Supérieur et de la Science (Action Structurante). 


\section{Abbreviations}

$T: \quad$ Transformation or shape model.

$T^{-1}: \quad$ The inverse transformation or model of $T$.

$T^{-1} T: \quad$ A cycle conversion of first the transformation $T$ and then its inverse transformation $T^{-1}$.

CSIM: The combined algorithm for $T^{-1} T$ by first using the splitting-shooting method in the $T$ procedure, and second using the splitting-integrating method in the $T^{-1}$ procedure.

CSSM: The combined algorithm for $T^{-1} T$ by using the splittingshooting method for both $T$ and $T^{-1}$ procedures.

CIIM: The combined algorithm for $T^{-1} T$ by using the splittingintegrating method in both $T$ and $T^{-1}$ procedures.

Eq.: Equation.

Eqs.: Equations.

Fig.: Figure.

Figs.: Figures.

2D: Two dimensions or two-dimensional.

3D: Three dimensions or three-dimensional. 


\section{Contents}

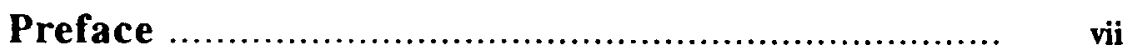

Acknowledgments …......................................... ix

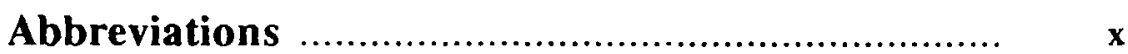

Chapter 1 Introduction ........................................ 1

Chapter 2 Linear Models ...................................... 7

1. Introduction ...................................................... 7

2. The Cartesian Coordinate Algorithm …..................... 9

3. The Area Coordinate Algorithm ............................. 12

4. Geometric Properties and Basic Transformations ......... 15

5. Error Analysis and Choice of Reference Triangles ....... 21

6. Linear Conformal Transformations ........................... 24

7. Recognition of Digitized Patterns .............................. 28

Chapter 3 Nonlinear Models …............................ 33

1. Introduction ..................................................... 33

2. Bilinear Models ................................................ 35

2.1. Algorithms ................................................. 36

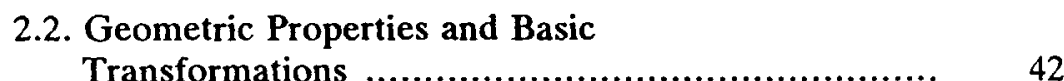


2.3. Error Analysis ..................................... 49

2.4. Inverse Transformations .............................. 51

3. Quadratic and Cubic Models .............................. 53

3.1. Quadratic Models ..................................... 54

3.2. Cubic Models ........................................... 58

4. Bi-quadratic and Bi-cubic Models ....................... 61

4.1. Bi-quadratic Models .................................. 61

4.2. Bi-cubic Models ...................................... $\quad 70$

5. The Coons Models ........................................ 74

6. Inverse Transformation Algorithms ..................... 78

7. Transformations in Three Dimensions ................... $\quad 79$

8. Perspective Transformations ............................ 87

Chapter 4 Discrete Techniques in Digitized

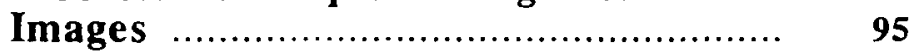

1. Introduction ................................................. 95

2. Splitting-Shooting Methods ............................. 97

2.1. Introduction ............................................. 97

2.2. A basic Splitting-Shooting Method ..................... 100

2.3. Splitting-Shooting Methods with Piecewise Linear Interpolation ..................................... 106

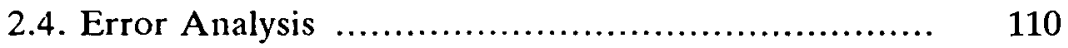

2.5. Numerical and Graphical Experiments ................ 122

3. Splitting-Integrating Methods ........................... 136

3.1. Introduction ........................................... 136

3.2. Description of the Methods and Error Bounds ....... $\quad 140$

3.3. Graphical Experiments ............................... 145

4. Combined Algorithms for a Cycle Conversion ............ 151

4.1. Introduction ........................................... 151

4.2. The Combined Algorithms ............................ 154

4.3. Applications of CSIM for Digital Images and Patterns ............................................ 157

Chapter 5 Combined Models, Harmonic Models and Comparisons of Models ............... 172

1. Combined Models ...................................... $\quad 172$ 
1.1. Addition of Models and Overlap of Images .......... 172

1.2. Subtraction of Models ............................... $\quad 176$

1.3. Sub-Models ........................................... 176

1.4. Coupling Models ....................................... 177

1.5. The Composite Models …............................. 178

2. Comparisons of Shape Models ........................... 179

3. Harmonic Models ........................................... 189

4. Mathematical-Physical Transformations .................... 198

\section{Chapter 6 Applications to Pattern Recognition} and Image Processing ................... 207

1. Normalization of Geometrical Shapes .................... 208

2. Recognition of Roman Letters and Chinese Characters ............................................... 216

3. A Practical Example of Chinese Character Recognition ........................................ 228

3.1. Description of the System ........................ 228

3.2. Analysis of Complex Texts ........................ 228

3.3. Design of the Recognition System .................. 232

3.4. Results of Experiments ............................. 233

4. Concluding Remarks ................................. 235

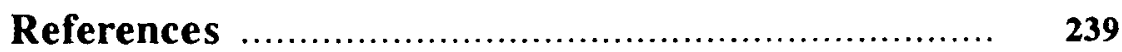

Glossary of Symbols .................................... 252

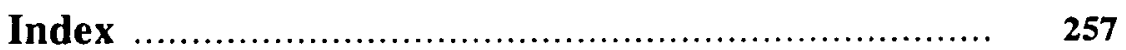

